\title{
Identification of characteristic molecular signature of Müllerian inhibiting substance in human HPV-related cervical cancer cells
}

\author{
SEONG JIN HWANG ${ }^{1}$, MIN JUNG SUH ${ }^{1}$, JOO HEE YOON ${ }^{1}$, MEE RAN KIM ${ }^{1}$, KI SUNG RYU ${ }^{1}$, \\ SUK WOO NAM ${ }^{2}$, PATRICIA K. DONAHOE ${ }^{3}$, DAVID T. MaCLAUGHLIN ${ }^{3}$ and JANG HEUB KIM ${ }^{1}$ \\ ${ }^{1}$ Department of Obstetrics and Gynecology, College of Medicine, The Catholic University of Korea; \\ ${ }^{2}$ Department of Pathology, Microdissection Genomics Research Institute, College of Medicine, \\ The Catholic University of Korea, Seoul, Republic of Korea; ${ }^{3}$ Pediatric Surgical Research Laboratories, \\ Massachusetts General Hospital and Harvard Medical School, Boston, MA 02114, USA
}

Received February 2, 2011; Accepted April 11, 2011

DOI: 10.3892/ijo.2011.1042

\begin{abstract}
Müllerian inhibiting substance (MIS), also known as anti-Müllerian hormone (AMH), is a member of the transforming growth factor- $\beta$ (TGF- $\beta$ ) superfamily that plays an important role in the mesenchymal-epithelial interaction, cell growth and proliferation, extracellular matrix production and tissue remodeling. Previously, we demonstrated that MIS suppressed ovarian cancer cell growth and suggested large-scale genetic elements that could be responsible for anti-neoplastic effects of MIS on ovarian cancer cells. In this study, we demonstrated the expression of MIS type II receptor (MISRII) in the human papillomavirus (HPV)-16-related cervical cancer cell lines CaSki and $\mathrm{SiHa}$, and a non-HPV-related cervical cancer cell line, C33A. We also showed that MIS inhibited growth of cervical cancer cells, and induced cellular apoptosis of C33A. In addition, we identified a characteristic molecular signature of MIS in CaSki cells by using whole genome expression analysis. Of the 1,690 genes that showed significant expression changes by MIS, 21 genes were related to cell cycle; 13 genes to apoptosis; and 52 genes to the cancer pathway. On performing a search for cell cycle pathways in the KEGG pathway database, several gene expressions at the $\mathrm{G}_{1} / \mathrm{S}$ checkpoint were found. In particular, the expression of p16 and p107 increased and that of E2F2 and E2F3 decreased at an early stage, whereas the expression of E2F4 and E2F5 decreased at a later stage after MIS treatment. These data suggest that MIS produces activity against HPV16-related cervical cancers in vitro, and MIS may also be an effective targeted therapy for HPV16-related cervical cancer. Genetic data obtained here could be useful in determining
\end{abstract}

Correspondence to: Professor Jang Heub Kim, Department of Obstetrics and Gynecology, College of Medicine, The Catholic University of Korea, \#505 Banpo-dong, Seocho-gu, Seoul 137-701, Republic of Korea

E-mail: janghkim@catholic.ac.kr

Key words: Müllerian inhibiting substance, anti-Müllerian hormone, Müllerian inhibiting substance type II receptor, CaSki cell line, DNA microarray, cell cycle the treatment strategy of MISR-expressing cervical tumors in the future.

\section{Introduction}

Müllerian inhibiting substance (MIS), also known as antiMüllerian hormone (AMH), is a glycoprotein composed of 2 identical subunits of 535 amino acids with a combined molecular weight of $140 \mathrm{kDa}$. It belongs to the TGF- $\beta$ family along with inhibin, activin, and bone morphogenetic protein (BMP) (1). An increasing number of studies have demonstrated that MIS plays key roles in sexual development as a hormone in the adult gonads of both sexes and in growth inhibition in cells expressing MIS receptors (MISRs) (2-4).

Because most gynecologic tumors originate from Müllerian duct, and MIS causes regression of the Müllerian duct, it is expected to inhibit growth of gynecologic cancer. Purified recombinant human MIS causes growth inhibition of epithelial ovarian cancer cells and cell lines in vivo and in vitro via MIS receptor-mediated mechanism (5-8). Further, several lines of evidence suggest that MIS inhibits growth in the tissue and cell line of other MIS receptor-expressed gynecologic malignancies such as cervical, endometrial, and breast cancers (9-11). It is known that the biological activity of MIS requires interaction with 2 similar but distinct receptors termed types I and II (MISRI, MISRII). Because MISRI is nearly ubiquitously expressed, the question of whether MISRI interacts with MISRII will depend on the tissue and/or species in consideration. MIS binding to its receptors initiates intracellular cascades that lead to the control of cell cycle regulating proteins and altered transcription of a number of genes, depending upon the target tissues. The MISRII gene is located on chromosome $12 \mathrm{q} 13$ and has 11 exons and 10 introns (12). The binding of MIS to MISRII leads to heterodimerization of receptors accompanied by a signaling cascade $(13,14)$. Activated MISRI initiates a signaling cascade of the intracellular Smad pathway originally found to be used in BMP downstream signaling. Besides the Smad pathway, there is evidence that MIS-mediated downstream effects are involved in the cell cycle. However, the mechanism underlying signal transduction is unclear in HPV-related cancer cell death after MIS treatment. 
In a previous study, it was found that both HPV16-related and non-HPV-related cervical cancer cell lines express the MIS type II receptor, which responds to MIS. Although MIS binds to both these cervical cancer cell lines, receptor expression has been rarely demonstrated (10); further, the functional consequences of this interaction are not well known. In Korea, cervical cancer is the most commonly diagnosed gynecologic malignancy. Most cervical cancers are exclusively related to HPV infection. Hence, the HPV16-related cervical cancer cell line CaSki was thought to be a suitable candidate for this study. Conventional genomic research tools demand a lot of time and effort. Therefore, DNA microarray technology is widely used for the genomic analysis of transcription levels. Many studies have presented the feasibility of this technique for identifying novel genes and for defining human cancers at the molecular level (15-17).

To better understand the molecular mechanisms by which MIS inhibits the growth of cervical cancer cells, we performed large-scale DNA microarray gene expression analysis and analyzed global transcript levels at different time points after treatment of HPV-related cervical cancer cells with MIS and investigated the characteristic genes that probably reflect the molecular signature for the anti-tumor effect of MIS (10).

\section{Materials and methods}

Recombinant human MIS. Recombinant human MIS was purified and its biological activity confirmed in the Pediatric Surgical Research Laboratories at the Massachusetts General Hospital from serum-free and serum containing conditioned media as described earlier (18).

Cells and cell culture. The HPV-transformed cervical cancer cell lines, CaSki and SiHa, and a non-HPV-transformed cervical cancer cell line, C33A and human kidney epithelial cell line 293 cells (ATCC, Manassas, VA, USA) served as a negative control were grown in RPMI-1640 and $15 \%$ female fetal bovine serum, $1 \%$ penicillin/streptomycin and $1 \% \mathrm{~L}$-glutamine for no more than 5 passages, and subcultures were initiated at $80 \%$ confluency. The cultures were maintained in a humidified atmosphere of $5 \% \mathrm{CO}_{2}$ at $37^{\circ} \mathrm{C}$.

Immunohistochemistry. Immunohistochemical staining for MISRII was visualized using a CAP-PLUS detection kit (Zymed Laboratories, South San Francisco, CA, USA). Briefly, 293, C33A, CaSki, and SiHa cells were harvested at $1 \times 10^{5} / \mathrm{ml}$ then $200 \mu 1$ cell suspension was centrifuged with Cytosin (Thermo Electron Corp., Cheshire, UK) at $1,000 \mathrm{rpm}$ for $5 \mathrm{~min}$ to attach cells to the Probe on plus slide. The slides were treated with $3 \% \mathrm{H}_{2} \mathrm{O}_{2}$ for $10 \mathrm{~min}$. After treatment with $5 \%$ goat serum, the slides were incubated with rabbit polyclonal anti-human MISRII antiserum (Pediatric Surgical Research Laboratories, Massachusetts General Hospital, Boston, MA, USA) at $4^{\circ} \mathrm{C}$ overnight. The slides were rinsed in PBS 3 times and incubated with biotinylated anti-rabbit IgG (Zymed Laboratories) at RT for $2 \mathrm{~h}$. After the PBS rinse, Streptavidin HRP detection system (Zymed Laboratories) was applied to the slides at RT for $30 \mathrm{~min}$. The slides were treated with 3-amino-9-ethylcarbazole (AEC) for $10 \mathrm{~min}$ at RT, counterstained with hematoxylin, and then mounted with glycerol gel.
Methylthiazoletetrazolium (MTT) assay. Three thousand 293, C33A, CaSki, and SiHa cells per well were seeded in 96-well plates. After $24 \mathrm{~h}$ the cells were exposed to vehicle control or $10 \mu \mathrm{g} / \mathrm{ml}$ of MIS for 24 and $48 \mathrm{~h}$ (19). Cells were washed with PBS and $100 \mu \mathrm{l}$ of MTT solution $(5 \mathrm{mg} / \mathrm{ml}$ MTT stock in PBS diluted to $1 \mathrm{mg} / \mathrm{ml}$ with $10 \%$ DMEM) was added to each well. Cells were incubated for $4 \mathrm{~h}$ at $37^{\circ} \mathrm{C}$ at the end of which time $200 \mu \mathrm{l}$ DMSO (Sigma, St. Louis, MO, USA) was added and incubated further for $30 \mathrm{~min}$ at room temperature in the dark. The plates were read on an ELISA plate reader (BIO-TEK Instruments, Winooski, VT, USA) and the absorbance at $550 \mathrm{~nm}$ was recorded. MIS-treated and -untreated cell lines were compared, and statistical analyses were performed by using the ANOVA paired t test, with $\mathrm{P}<0.05$ considered to be statistically significant.

Cell cycle analysis. 293, C33A, CaSki, and SiHa cell lines were exposed to MIS or vehicle control buffer for $24 \mathrm{~h}$ and the cells were collected by trypsinization. The cells were fixed with $100 \%$ methanol and stored for $30 \mathrm{~min}$ at $20^{\circ} \mathrm{C}$ and washed with PBS. Following centrifugation the cells were resuspended in $1 \mathrm{ml} \mathrm{DNA} \mathrm{staining} \mathrm{solution}(20 \mu \mathrm{g} / \mathrm{ml}$ propidium iodide, $200 \mu \mathrm{g} / \mathrm{ml}$ DNase free RNase) and incubated in the dark at $37^{\circ} \mathrm{C}$ for $30 \mathrm{~min}$. The cells were analyzed on a FACSVantage SE Flow Cytometer (Becton-Dickinson, San Jose, CA, USA). The forward scatter and red fluorescence above $600 \mathrm{~nm}$ were measured and the results analyzed using Cell Quest ${ }^{\mathrm{TM}}$ software (Becton-Dickinson) and Modfit LT 3.0 program (Verity Software House, Topsham, ME, USA).

Annexin $V$ analysis. MIS-treated cells were stained for Annexin $\mathrm{V}$ and propidium iodide (PI) using the Annexin VFITC Apoptosis Detection Kit I (BD Biosciences, San Diego, CA, USA) according to the manufacturer's protocol. Briefly, following drug treatment, $1 \times 10^{5}$ cells were pelleted and washed once with PBS and resuspended in $100 \mathrm{ml}$ of binding buffer [10 mM HEPES (pH 7.4), $150 \mathrm{mM} \mathrm{NaCl}, 5 \mathrm{mM}$ potassium chloride, $1 \mathrm{mM} \mathrm{MgCl}_{2}$, and $2 \mathrm{mM}$ calcium chloride]. Subsequently, $5 \mu \mathrm{l}$ of Annexin V-FITC and PI was added to the cells that were then incubated for $15 \mathrm{~min}$ at RT in the dark. After this incubation, $400 \mu \mathrm{l}$ of binding buffer was added and cells were analyzed using a FACSVantage SE flow cytometer (Becton-Dickinson). Data analyses were conducted using Cell Quest $^{\mathrm{TM}}$ software (Becton-Dickinson).

Microarray fabrication. The 60-mers of Human Oligolibrary ${ }^{\mathrm{TM}}$ representing 18,664 LEADS ${ }^{\mathrm{TM}}$ clusters plus 197 controls (GAPDH) were purchased from Compugen/Sigma-Genosys and spotted onto a glass slide at the microarray core facility of Microdissection Genomics Research Institute at College of Medicine, The Catholic University of Korea as previously reported $(20,21)$.

Large-scale analysis of gene expression profiling by using oligonucleotide arrays. In brief, total cellular RNA was extracted from the vehicle control and MIS-treated cells using a TRIzol ${ }^{\circledR}$ reagent (Gibco, Grand Island, NY, USA) and quantified using NanoDrop (NanoDrop Technologies, Wilmington, DE, USA). In addition, RNA quality was checked by Bioanalyzer 2100 (Agilent Technologies, Santa Clara, CA, USA) and intact RNA 
A

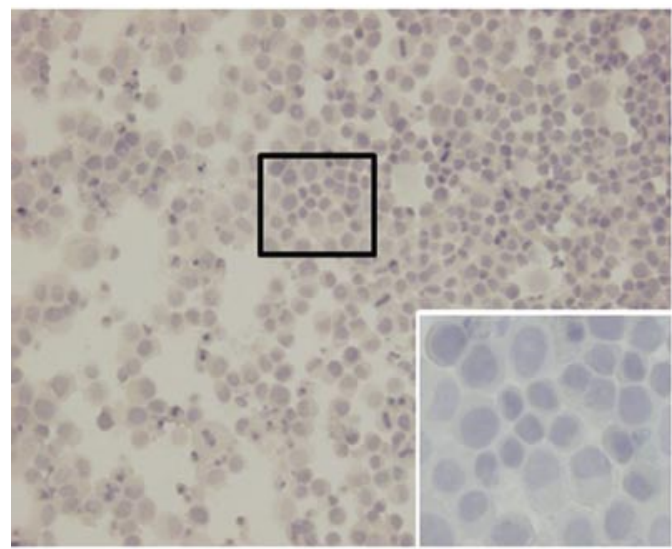

C

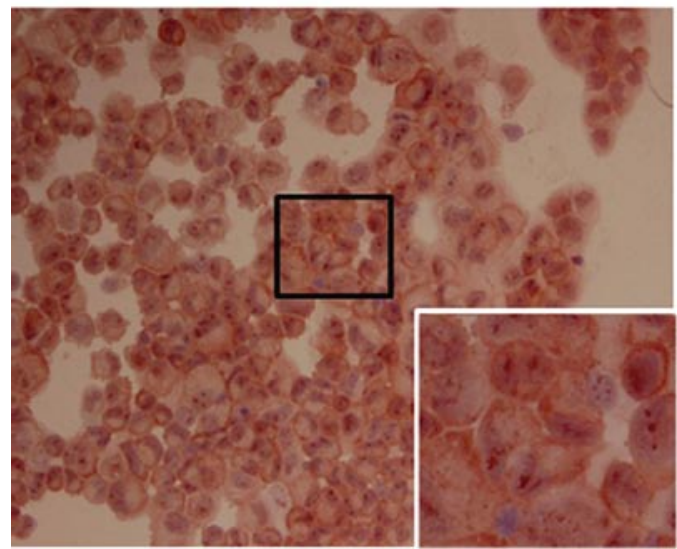

B

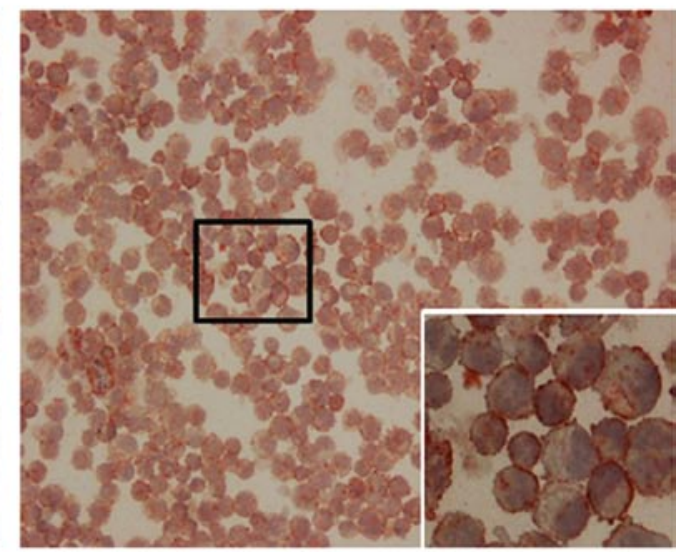

D

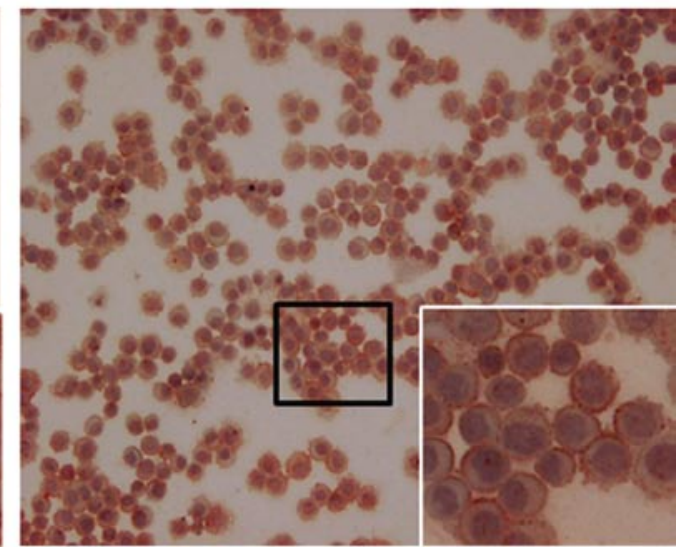

Figure 1. Immunohistochemistry using MISRII antibody from the human kidney epithelial cell line, 293 cells (A) and cervical cancer cell line, C33A (B), CaSki (C), and SiHa cells (D). Compared to 293 cell line showing negative staining pattern for MISRII, all cervical cancer cell lines showed strong specific staining for MISRII; staining in C33A was the most intense (x200). At higher magnification (x400) of the boxed area of (B, C, and D), MISRII was localized specifically and exclusively in the cell membrane and occasionally in the Golgi's complex of the cytoplasm. The nucleus does not stain for MISRII.

samples $(260 / 280$ ratio $>1.8)$ were used for hybridization. Human universal reference RNA (Stratagene, La Jolla, CA, USA) was used as a reference RNA. From each total RNA sample, $20 \mu \mathrm{g}$ was primed with oligo-dT and labeled with Cy3-dUTP or Cy5-dUTP (New England Nuclear, Wilmington, MA, USA) by reverse transcription. Labeled cDNA targets were concentrated to approximately $15 \mu \mathrm{l}$ using Microcon-YM30 (Amicon, Bedford, MA, USA). Hybridization was carried out at $42^{\circ} \mathrm{C}$ for $16 \mathrm{~h}$ using the MAUI Hybridization System (BioMicro Systems, Salt Lake City, UT, USA). After hybridization, microarray was washed twice with buffers containing $2 \mathrm{X}$ standard sodium citrate (SSC) and $0.1 \%$ SDS for $2 \mathrm{~min}, 1 \mathrm{X}$ SSC and $0.1 \%$ SDS for $3 \mathrm{~min}, 0.2 \mathrm{X} \mathrm{SSC}$ for $3 \mathrm{~min}, 0.05 \mathrm{X} \mathrm{SSC}$ for $2 \mathrm{~min}$, and finally rinsed with distilled water for $2 \mathrm{~min}$. This protocol was repeated twice in independent experiments on the CaSki cells.

Scanning and data analysis. The arrays with hybridized targets were scanned using an Axon scanner and the images were analyzed using GenePix ${ }^{\circledR}$ Pro 4.1 software (Molecular Devices, Sunnyvale, CA, USA). Spots of poor quality identified by visual inspection were removed from further analysis. The resulting data collected from each array were submitted to the BioArray Software Environment (BASE) database at the microarray core facility in the Department of Pathology, College of
Medicine at The Catholic University of Korea, Seoul, Republic of Korea (http://genomics.catholic.ac.kr/). Data were normalized using the method of Linear Models for Microarray Data (LIMMA) and R-package for Statistics for Microarray Analysis (SMA). Spots of less than $50 \mu \mathrm{m}$ were eliminated for analysis unless otherwise specified. Pearson's correlation coefficient was calculated using S-PLUS program. Cluster and TreeView programs were used for visualization of data $(17,20)$. Pathway analysis was performed with ArrayXPath (http:// www.snubi.org/software/ArrayXPath/). The statistical analysis was performed by using the Mann-Whitney test, with $\mathrm{P}<0.05$ considered to be statistically significant.

\section{Results}

Expression of MISRII by immunohistochemistry. Compared to the negative control 293 cell line showing negative staining pattern for MISRII, all three cervical cancer cell lines showed strong specific staining for MISRII by using immunohistochemical staining, although staining in C33A cells was more intense than that in CaSki and SiHa. MISRII was localized specifically and exclusively in the cell membrane and occasionally in the Golgi's complex of the cytoplasm of cervical cancer cells (Fig. 1). 

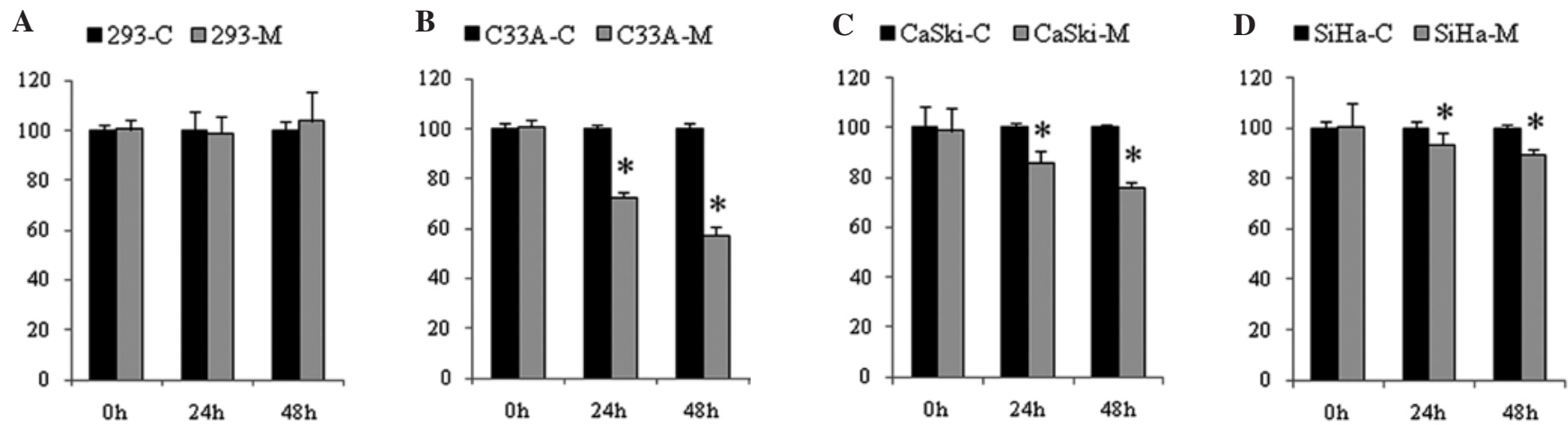

Figure 2. Effect of MIS on the viability of 293 (A) and of C33A (B), CaSki (C), and SiHa cells (D). Cells were treated with MIS at $10 \mu \mathrm{g} / \mathrm{ml} 24 \mathrm{~h}$ after plating. At 24 and $48 \mathrm{~h}$ after treatment, cells were stained MTT, and the absorbance was read at $550 \mathrm{~nm}$. Results were presented as percentage of control which was calculated using the equation: (mean absorbance of treated cells/mean absorbance of control cells) x 100. Data were expressed as mean \pm standard deviation (SD) from three independent experiments. $* \mathrm{P}<0.05$ as compared to corresponding control cells.

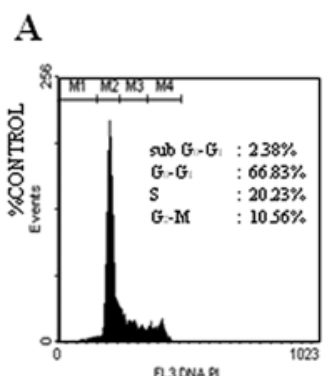

B

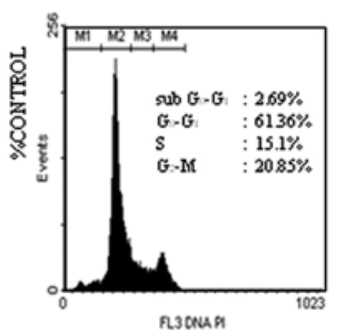

$\mathrm{Oh}$

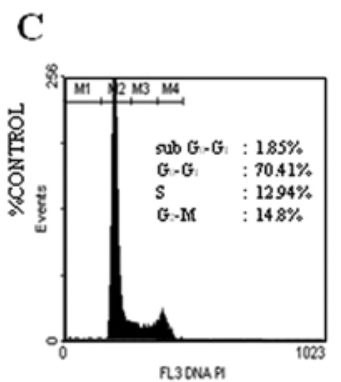

$\mathrm{Oh}$

D

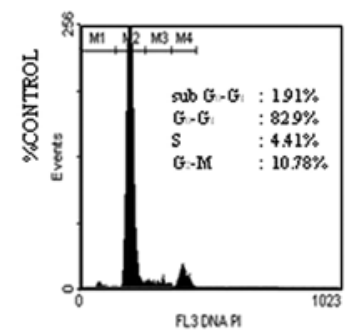

$\mathrm{Oh}$

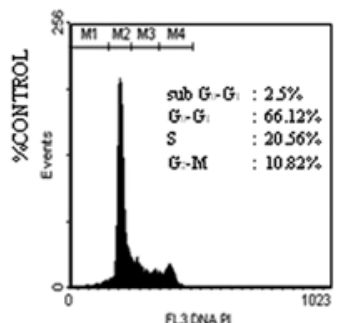

$24 \mathrm{~h}$

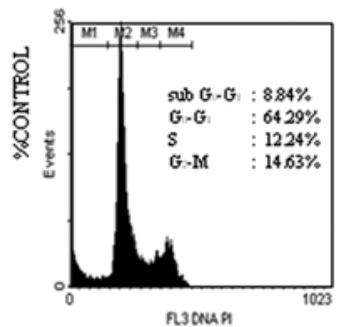

$24 \mathrm{~h}$

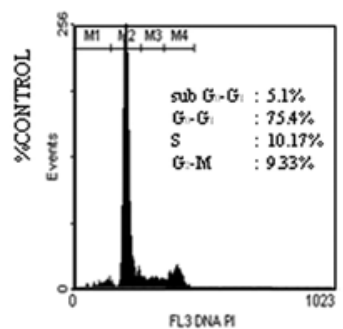

$24 \mathrm{~h}$

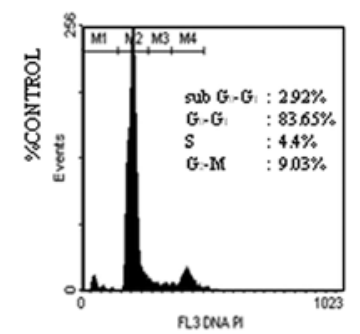

$24 \mathrm{~h}$

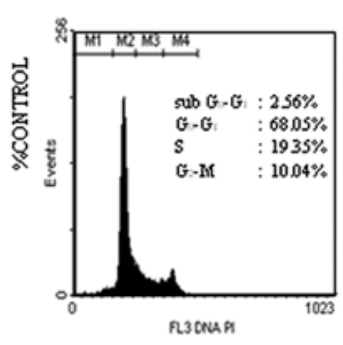

$48 \mathrm{~h}$

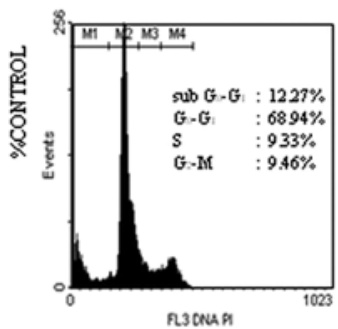

$48 \mathrm{~h}$

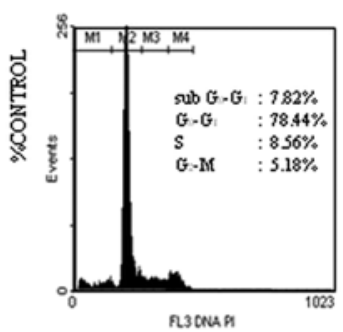

$48 \mathrm{~h}$

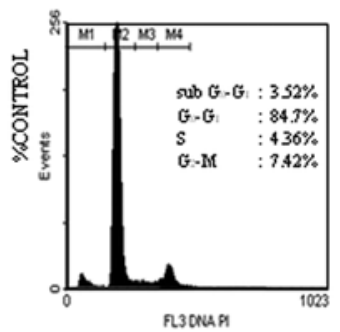

$48 \mathrm{~h}$

Figure 3. Cell cycle distribution after exposure to MIS in 293 (A) and of C33A (B), CaSki (C), and SiHa cells (D). Cells were treated with $10 \mu \mathrm{g} / \mathrm{ml}$ MIS for 24 and $48 \mathrm{~h}$, respectively. Then they were trypsinized and fixed in $70 \%$ ethanol. After washing, cells were exposed to propidium iodide/RNase solution. Histograms of cellular DNA content were obtained by flow cytometry. 

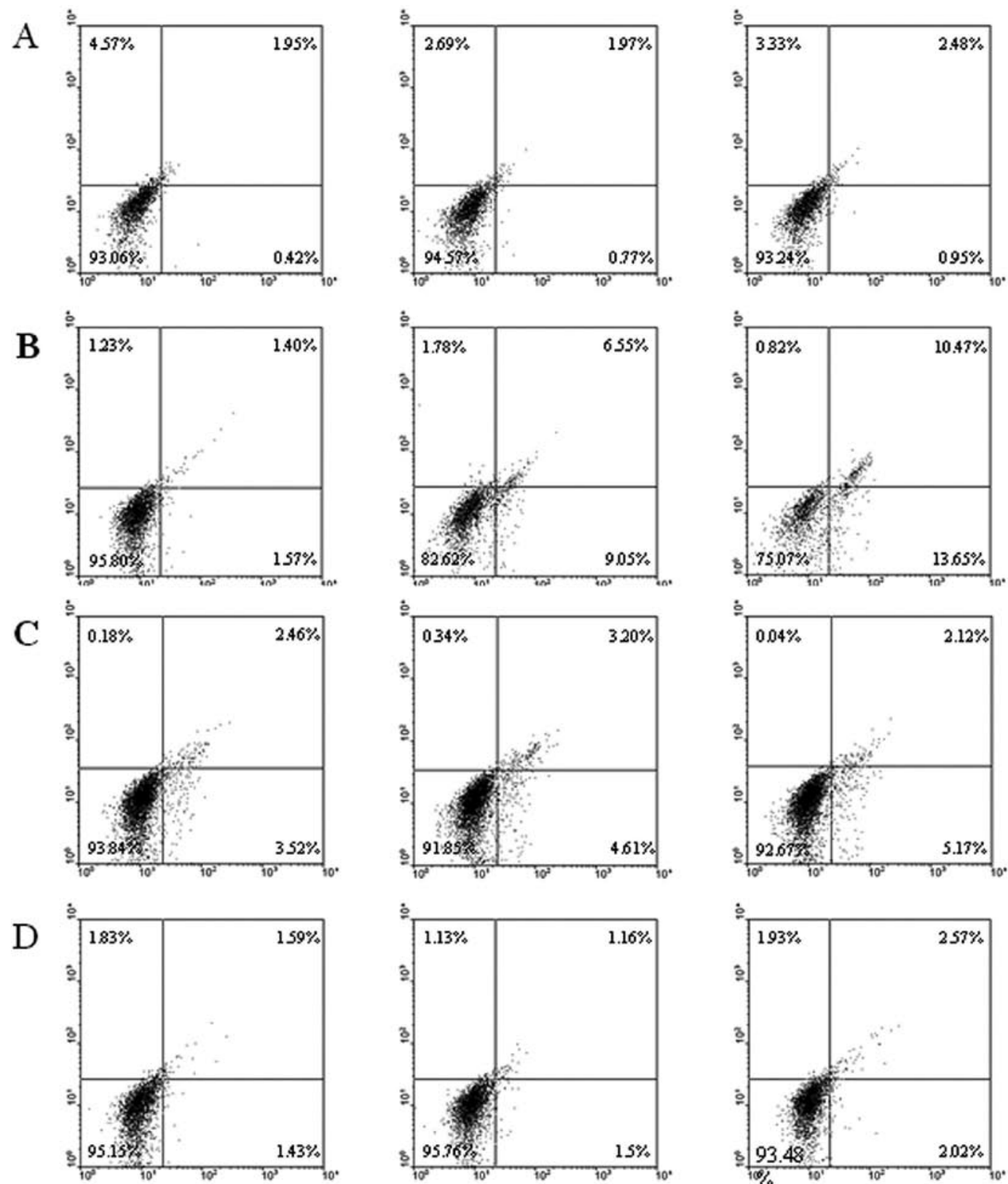

$\mathrm{Oh}$

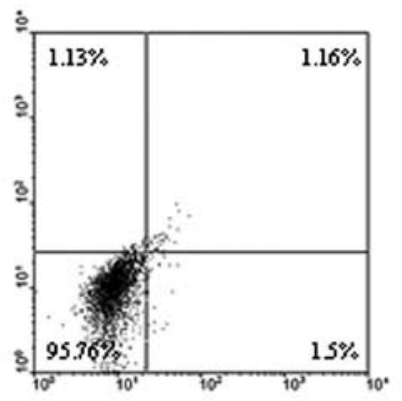

$24 \mathrm{~h}$

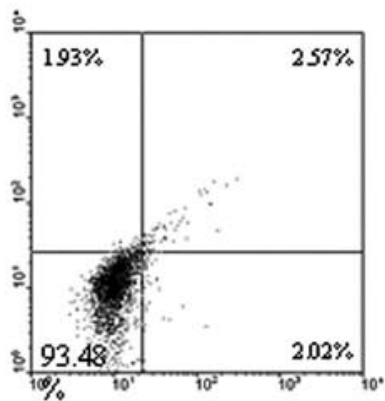

$48 \mathrm{~h}$

Figure 4. Induction of appoptosis by MIS in 293 (A) and of C33A (B), CaSki (C), and SiHa cells (D). Cells were treated with $10 \mu \mathrm{g} / \mathrm{ml} \mathrm{MIS}$ for 24 and $48 \mathrm{~h}$, respectively. For apoptosis, the externalization of phosphatidylserine was assessed by measuring Annexin-V-FITC binding using propidium iodide as a counterstain. Quadrant rectangular dot grams from a representative of 3 independent experiments are shown.

MIS inhibits the growth of cervical cancer cells and augments apoptosis. The MTT assay was used to quantify the degree of growth suppression. As predicted, MIS had no effect on inhibition of 293 cells, which do not express the MISRII (Fig. 2). Growth inhibition of MISRII-expressed cervical cancer cells by MIS was evident at both 24 and 48 h. As shown in Fig. 2, the cell viability was decreased to $57-72 \%$ of control in $\mathrm{C} 33 \mathrm{~A}$ cell, 76-85\% in CaSki cell, and 89-93\% in SiHa cell. C33A and CaSki cells showed the negative correlation between the MIS exposure time and the viability $(\mathrm{P}<0.05)$.

To determine whether the anti-growth activity was related to cell cycle regulation, the cell cycle phase distribution after MIS treatment was analyzed by flow cytometry (Fig. 3). When cells were incubated with $10 \mu \mathrm{g} / \mathrm{ml}$ of MIS for 24 and $48 \mathrm{~h}$,
S and $G_{2} M$ phases were decreased, and sub- $G_{0} G_{1}$ and $G_{0} G_{1}$ phases were increased. In C33A cell, the sub- $\mathrm{G}_{0} \mathrm{G}_{1}$ and $\mathrm{G}_{0} \mathrm{G}_{1}$ phases were increased $6 \%$ and $3 \%$ at $24 \mathrm{~h}$ and $10 \%$ and $8 \%$ at $48 \mathrm{~h}$ compared to controls, respectively. In CaSki cell, the sub- $\mathrm{G}_{0} \mathrm{G}_{1}$ and $\mathrm{G}_{0} \mathrm{G}_{1}$ phase was increased $3 \%$ and $5 \%$ at $24 \mathrm{~h}$ and $6 \%$ and $8 \%$ at $48 \mathrm{~h}$ compared to controls, respectively, but $\mathrm{SiHa}$ cell demonstrated minimal response. This finding could be partially explained by the fact that MIS may induce $\mathrm{G}_{1} / \mathrm{S}$ arrest at an early time point subsequently leading to celldeath.

In fact, time-related induction of cell death by MIS on cervical cancer cell lines was detected by FACS analysis of cells stained with Annexin V-FITC and PI (Fig. 4). Annexin VFITC staining showed cellular apoptosis to be $9.05 \%, 4.61 \%$ 
A
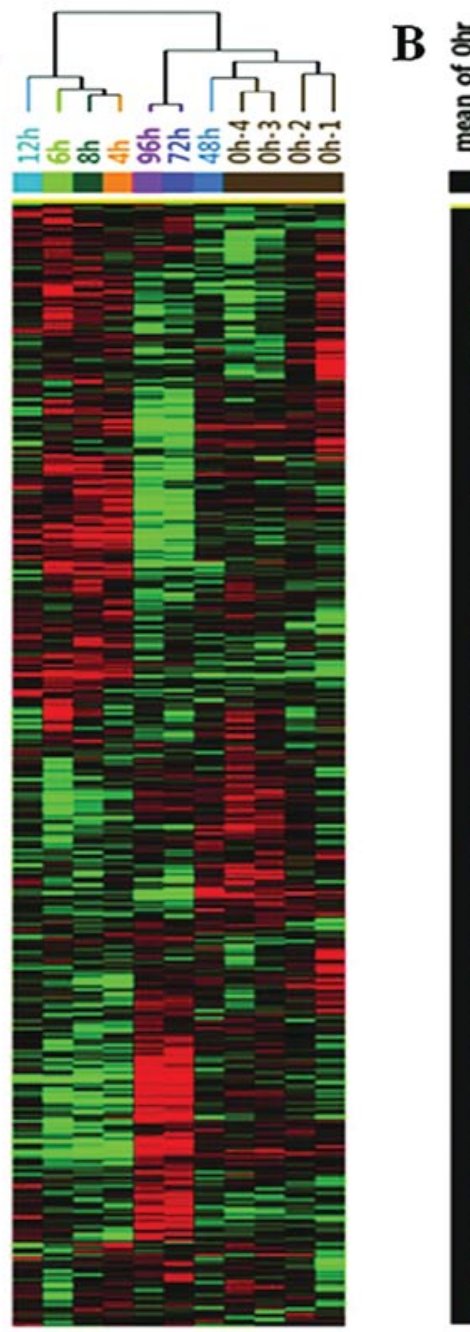

B

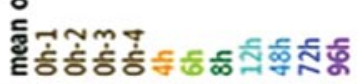$$
\text { E }
$$

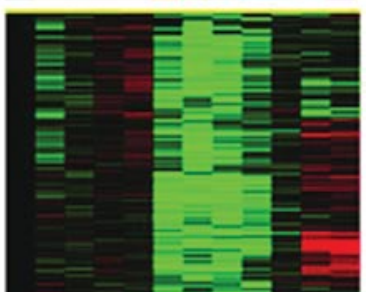

Table I. Summary of the KEGG pathways that are part of the characteristic molecular signature of MIS in Caski cells.

\begin{tabular}{|c|c|}
\hline KEGG pathway & No. of genes \\
\hline Pathways in cancer & 52 \\
\hline Focal adhesion & 37 \\
\hline MAPK signaling pathway & 32 \\
\hline Small cell lung cancer & 25 \\
\hline Regulation of actin cytoskeleton & 25 \\
\hline Cytokine-cytokine receptor interaction & 25 \\
\hline Jak-STAT signaling pathway & 23 \\
\hline p53 signaling pathway & 22 \\
\hline Ubiquitin mediated proteolysis & 21 \\
\hline Systemic lupus erythematosus & 21 \\
\hline Cell cycle & 21 \\
\hline ECM-receptor interaction & 21 \\
\hline Tight junction & 17 \\
\hline Wnt signaling pathway & 17 \\
\hline Pyrimidine metabolism & 17 \\
\hline Purine metabolism & 16 \\
\hline Pathogenic Escherichia coli infection-EPEC & 16 \\
\hline Pathogenic Escherichia coli infection-EHEC & 16 \\
\hline Antigen processing and presentation & 15 \\
\hline Axon guidance & 15 \\
\hline TGF- $\beta$ signaling pathway & 15 \\
\hline Pancreatic cancer & 14 \\
\hline Huntington's disease & 14 \\
\hline Bladder cancer & 13 \\
\hline Apoptosis & 13 \\
\hline Leukocyte transendothelial migration & 13 \\
\hline Neuroactive ligand-receptor interaction & 13 \\
\hline Cell adhesion molecules (CAMs) & 12 \\
\hline Colorectal cancer & 12 \\
\hline Calcium signaling pathway & 12 \\
\hline Hematopoietic cell lineage & 11 \\
\hline Cardiac muscle contraction & 11 \\
\hline Prostate cancer & 11 \\
\hline PPAR signaling pathway & 11 \\
\hline Insulin signaling pathway & 11 \\
\hline Glycolysis/gluconeogenesis & 10 \\
\hline Alzheimer's disease & 10 \\
\hline Parkinson's disease & 10 \\
\hline Biosynthesis of steroids & 10 \\
\hline Renal cell carcinoma & 10 \\
\hline Chronic myeloid leukemia & 10 \\
\hline Natural killer cell mediated cytotoxicity & 10 \\
\hline
\end{tabular}

There were significant pathways including more than 10 genes by one pathway in outlier of 1,690 genes which showed expression more than 1.5 -fold increase or decrease. signature that is specifically induced by MIS, serial gene expression analyses using large scale comprehensive DNA microarray experiments were conducted. Because MIS showed sustained growth inhibitory activity for at least $48 \mathrm{~h}$, CaSki cells were continuously treated with MIS for up to $96 \mathrm{~h}$ and the DNA harvested for microarray analysis at multiple time points

Figure 5. Differential gene expression profiling and identification of large-scale molecular changes in the CaSki cervical cancer cell line that was treated with MIS at $10 \mu \mathrm{g} / \mathrm{ml}$. (A), Unsupervised hierarchical clustering of 14,930 genetic elements with minimum selection and filtering criteria resulted in two divided subcultures on dendrogram. (B), A significant subset of outlier genes was further narrowed by filtering genes showing expression changes due to MIS treatment of cells. Briefly, the mathematical comparisons of genes between 0 and the 4- to 96-h time points were performed by selection of genes showing at least 1.5 -fold changes induced by MIS treatment compared to non-treated control $(0 \mathrm{~h})$. This analysis resulted in the identification of 1,690 outlier gene elements were then visualized as a heat map where the red color indicates that expression levels of genetic elements are higher than the mean value of non-treatment, and green color indicates that expression levels of genetic elements are lower than the mean value of non-treatment.

and $1.50 \%$ after $24 \mathrm{~h}$ of MIS exposure and $13.65 \%, 5.17 \%$ and $2.02 \%$ after $48 \mathrm{~h}$ in C33A, CaSki and $\mathrm{SiHa}$, respectively. There was a negative correlation between cellular survival and MIS exposure time. Identification of large-scale molecular changes and chara-
cteristic molecular signature of MIS. To identify a molecular A

as early as 6 h. As shown in Fig. 5A, unsupervised hierarchical clustering of 14,930 genetic elements, which passed the 

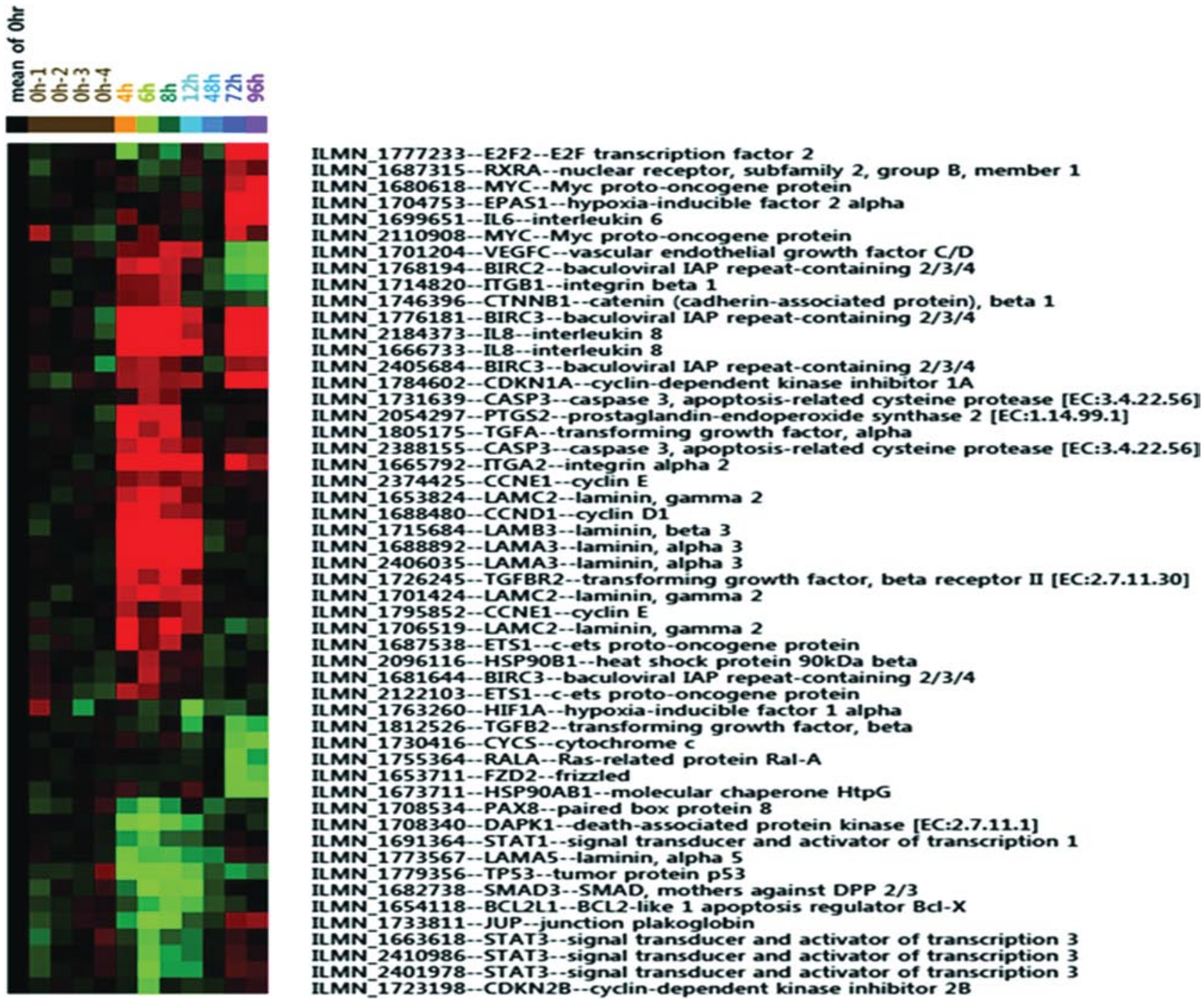

Figure 6. Outlier expression profile of cancer pathway-related genetic elements changed significantly by MIS in the CaSki cells. Of 1,690 outlier genes, 52 genes were mapped into pathway in cancer which included most genetic elements (Table I) using the KEGG pathway database. The expression patterns of genes were then visualized as a heat map. The red color indicates that expression levels of genetic elements are higher than the mean value of non-treatment, and green color indicates that expression levels of genetic elements are lower than mean value of non-treatment.

minimum selection, and filtering criteria (Materials and methods) resulted in two separate subclusters on the dendrogram; a short-term exposure group (0-24 h of MIS-treatment) and a long-term exposure (48-96 h) group. To identify genes that are continuously changing due to MIS action, mean values of the entire pool of genetic elements in non-treated cells $(0 \mathrm{~h}$ of MIS) was subtracted from that of the long-term exposure group. The 1690-outlier genes from this analysis were then re-examined in the expression data of short-term exposure group and then shown as a heat map (Fig. 5B). As shown in this figure, most of outlier genes showed gradual and continuous changes from early MIS exposure with a few exceptions (Fig. 5B).

Molecular dissection of cell growth regulation by MIS through pathway mining analyses. By an in depth examination of the genes identified as candidate MIS targets it was possible to locate downstream signaling pathways under the control of long-term MIS treatment of CaSki cells. This process was accomplished by subjecting the MIS regulated 1,690 genes to the pathway mining tool in PANTHER Classification System (http://www.pantherdb.org/). As listed in Table I, major processes that MIS-characteristic genes are involved in are pathways in cancer, cell cycle, and apoptosis. Out of 14,930 genes showing time-dependent change (Fig. 5A), only 1,690 genes showed differences of expression more than 1.5-fold and were grouped as functional pathway category (Fig. 5B).

Of 1,690 outlier genes, 52 genes were mapped into cancer pathways which included most genetic elements (Table I) using the KEGG pathway database (Fig. 6). Genes $(n=21)$ were mapped into cell cycle (Fig. 7) and 13 genes were mapped into apoptosis (Fig. 8). MIS treatment elevated the expression of p107 and p16, especially variant 1 among the 3 transcript variants. In contrast, expression of p130 showed no significant change. Interestingly, an increase in the expression of p107 and a decrease in the expression of E2F2 and E2F3 were observed after MIS treatment (Fig. 9). The expression of E2F4 and E2F5 showed a decrease in late time point, in contrast, E2F6, E2F7 showed an increase $72 \mathrm{~h}$ and at $96 \mathrm{~h}$ after MIS treatment respectively. 


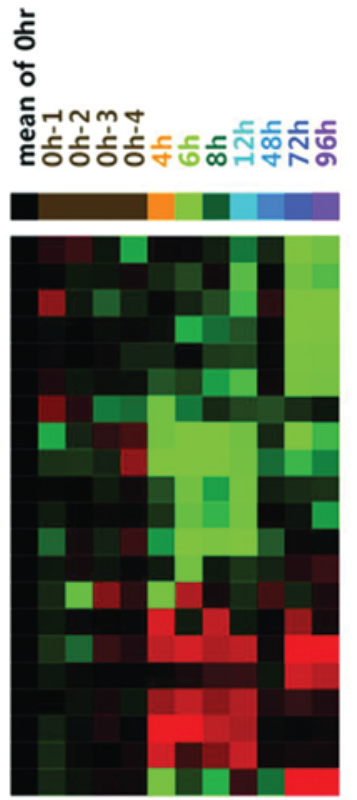

ILMN_2202948--BUB1--checkpoint serine/threonine-protein kinase [EC:2.7.11.1]

ILMN 1812526--TGFB2--transforming growth factor, beta

ILMN_1712803--CCNB1--cyclin B

ILMN_1753196--PTTG1--pituitary tumor-transforming

ILMN-1736176--PLK1--polo-like kinase 1 [EC:2.7.11.21]

ILMN_1801939--CCNB2--cyclin B

ILMN_2386100--BUB3---ceी cycle arrest protein BUB3

ILMN_1806607--SFN--stratifin

ILMN-1779356--TP53--tumor protein p53

ILMN_2407619--CDC25C--cell division cycle 25C [EC:3.1.3.48]

ILMN_1748883--CDKN2D--cyclin-dependent kinase inhibitor 2D

ILMN 1682738--SMAD3--SMAD, mothers against DPP 2/3

ILMN 1723198--CDKN2B--cyclin-dependent kinase inhibitor 2B

ILMN-1704702--MCM7--minichromosome maintenance protein 7 (cell division control protein)

ILMN-1694075--GADD45A--growth arrest and DNA-damage-inducible protein

ILMN_1784602--CDKN1A--cyclin-dependent kinase inhibitor $1 A$

ILMN 1762526--SMC1B--structural maintenance of chromosome 1

ILMN_2374425--CCNE1--cyclin E

ILMN-1688480--CCND1--cyclin D1

ILMN-1795852--CCNE1--cyclin E

ILMN_1777233--E2F2--E2F transcription factor 2

Figure 7. Outlier expression profile of cell cycle-related genetic elements changed significantly by MIS in the CaSki cells. Of 1,690 outlier genes, 21 genes were mapped into cell cycle through the KEGG pathway database.

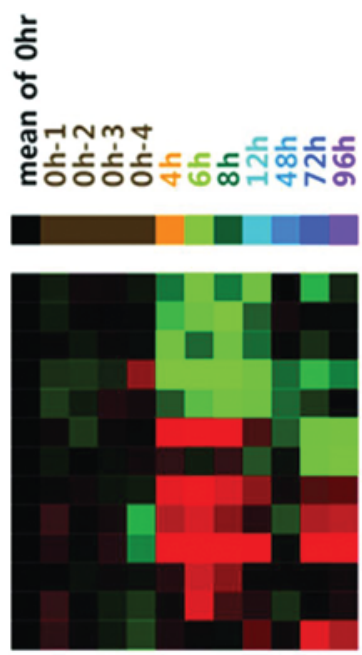

ILMN_1775501--IL1B--interleukin 1, beta

ILMN 1765851--TRADD--tnfrsfla-associated via death domain

ILMN_1705261--CAPN1--calpain-1 [EC:3.4.22.52]

ILMN_1779356--TP53--tumor protein p53

ILMN_1654118--BCL2L1--BCL2-like 1 apoptosis regulator $\mathrm{Bcl}-\mathrm{X}$

ILMN_1768194--BIRC2--baculoviral IAP repeat-containing 2/3/4

ILMN-1730416--CYCS--cytochrome c

ILMN_2388155--CASP3--caspase 3, apoptosis-related cysteine protease [EC:3.4.22.56]

ILMN 2405684--BIRC3--baculoviral IAP repeat-containing 2/3/4

ILMN_1776181--BIRC3--baculoviral IAP repeat-containing 2/3/4

ILMN_1731639--CASP3--caspase 3, apoptosis-related cysteine protease [EC:3.4.22.56]

ILMN_1681644--BIRC3--baculoviral IAP repeat-containing 2/3/4

ILMN_1699265--TNFRSF10B--tumor necrosis factor receptor superfamily, member 10

Figure 8. Outlier expression profile of apoptosis genetic elements changed significantly by MIS in the CaSki cells. Of 1,690 outlier genes, 13 were mapped to apoptotic pathways in the KEGG database.

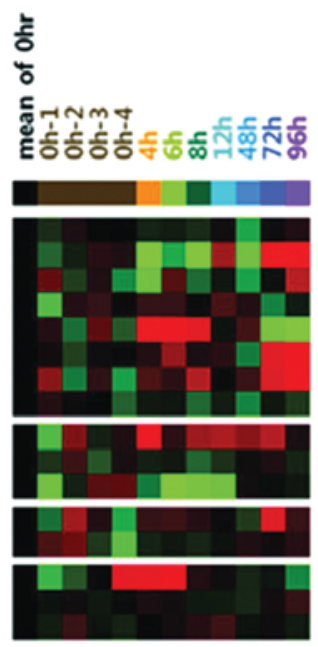

E2F1--E2F transcription factor 1 E2F1

E2F2--E2F transcription factor 2 E2F2

E2F3--E2F transcription factor 3 E2F3

E2F4--E2F transcription factor 4, p107/p130-binding E2F4

E2F5--E2F transcription factor 5 , p130-binding E2F5, transcript variant 1

E2F6--E2F transcription factor 6 E2F6

E2F7--E2F transcription factor 7 E2F

E2F8--E2F transcription factor 8 E2F8

CDKN2A--cyclin-dependent kinase inhibitor 2A (melanoma, p16, inhibits CDK4) (CDKN2A), transcript variant 1

CDKN2A--cyclin-dependent kinase inhibitor 2A (melanoma, p16, inhibits CDK4) (CDKN2A), transcript variant 3

CDKN2A-cyclin-dependent kinase inhibitor 2A (melanoma, p16, inhibits CDK4) (CDKN2A), transcript variant 4

CDKN1B--cyclin-dependent kinase inhibitor 1B p27, Kip1 CDKN1B

CDKN1B--cyclin-dependent kinase inhibitor 1B p27, Kip1 CDKN1B

RBL1--retinoblastoma-like 1 p107 RBL1, transcript variant 1

RBL1--retinoblastoma-like 1 (p107) (RBL1), transcript variant 2

RBL2--retinoblastoma-like 2 p130 RBL2 


\section{Discussion}

In a previous study with non-HPV-related cervical cancer cell line C33A, MIS treatment induced up-regulation of both p107 and p130 proteins; however, the functional consequence of this interaction has not been reported in the HPV-related cervical cancer cell line CaSki thus far (10).

It has also been demonstrated in previous studies that recombinant human MIS inhibits the growth of the human epithelial ovarian cancer cell line OVCAR-8 through specific induction of the cyclin-dependent kinase inhibitor (CDKI) p16 expression and via regulation of p130 and E2F1 when Rb levels were not detectable (5). A similar mode of cell cycle regulation by MIS has been investigated in other cancers $(10,11)$. In the non-HPV-related human cervical cancer cell line C33A, MIS induces expression of tumor suppressor protein p16. In addition to the induction of p16 expression, the up-regulation of p107, p130, and E2F1 is important to MIS for arresting cell growth. This finding suggested that MIS needs p130 for cell cycle exit and p107 for growth inhibition in cervical cancer cells, which in turn arrest cell growth at the $\mathrm{G}_{1} / \mathrm{S}$ phase (10).

Cervical cancer remains the second most common cause of cancer-related deaths in women worldwide. An important factor in cervical cancer is the infection of high-risk HPV. HPVs are causative agents of over $99 \%$ of cervical cancers. Mutations in cellular genes and chromosomal rearrangements induced by genomic instabilities are important contributing events (21). Hence, in order to determine whether cervical cancer is sensitive to MIS, we investigated the effect of MIS on HPV-transformed cervical cancer cell lines.

In this study, to reveal the molecular mechanism by which MIS inhibits the growth of cervical cancer cells, we analyzed the HPV16-related human cervical cancer cell line CaSki, which expresses significant levels of MISRII. For the sake of elucidating mechanisms by which MIS affects the growth of cervical cancer cells of a more common type of cervical cancer, in terms of HPV status, we examined the CaSki cell line using the DNA microarray technique to get a broad insight into the process. CaSki cells related to HPV-16 showed $15-24 \%$ reduction in cell survival and an increase in $\mathrm{G}_{0} \mathrm{G}_{1} /$ sub- $\mathrm{G}_{0} \mathrm{G}_{1}$ along with decrease in $\mathrm{S}$ phase subfraction. These results were supported by microarray data adapted to pathway analysis for cell cycle; the data showed that many of the genes whose expression increased greater than 1.5 -fold were those that are well known to be responsible for the $\mathrm{G}_{1} / \mathrm{S}$ checkpoint. In this experiment, we found that after MIS treatment, the expression levels of E2F2 and E2F3 decreased, whereas that of E2F1 showed no significant change. It implies that E2F2 and E2F3 are more likely than E2F1 to be associated with MIS-induced apoptosis of HPV16-related cervical cancer cells.

It has been reported that in many types of cells, MIS inhibits proliferation by increasing the expression of $\mathrm{p} 16$, a protein of the INK4 family of CDKIs, and adjusting the expression levels of p107 and p130, which act as $\mathrm{G}_{1}$ checkpoints in a pRbindependent manner, and then finally increasing the expression of the transcription factor E2F, especially that of E2F1 and E2F4, in normal ovarian cortex cells (HOSE6-3), the ovarian cancer cell line OVCAR-8, and the cervical cancer cell C33A with a pRb mutation $(5,10)$. On the other hand, in HPV-positive CaSki and SiHa cells, the HPV E7 protein binds to the $\mathrm{pRb}$ protein family, including proteins p107 and p130 (5). However, considering the unusual overexpression of $\mathrm{p} 16^{\mathrm{INK} 4 \mathrm{~A}}$ in cells showing pRB inactivation by HPV (22), an antiproliferative mechanism similar to that in $\mathrm{C} 33 \mathrm{~A}$ cells could be assumed for CaSki cells, even though the degree of suppression differs. Even in endometrial cancer cell lines (AN3CA) lacking p16 and pRb, MIS-induced apoptosis by increasing p107 and p130 expression in a p16-independent manner and decreasing E2F1 expression (11).

CaSki cells show a persistently low expression of $\mathrm{pRb}$; hence, we cannot exclude the possibility that $\mathrm{pRb}$ may be contributing in some minor way in cell cycle control. MIS treatment elevated the expression of p16, which is known to arrest the cell cycle by blocking CDK4/6, especially variant 1 among the 3 transcript variants. Interestingly, an increase in the expression of $\mathrm{p} 107$, which is a pRb-related pocket protein, and a decrease in the expression of E2F2 and E2F3 were observed after MIS treatment. Cell cycle arrest induced by p16 is known to be mediated by p107 and p130, and pRb was found to be insufficient for a p16-induced cell cycle arrest in primary mouse embryonic fibroblasts specifically lacking pRb-family members $(23,24)$.

As in the ovarian cancer cell line OVCAR-8, growth suppression in the C33A cervical cancer cell line by MIS is associated with induction of the p16 tumor suppressor protein. However, in contrast to OVCAR-8 cells, induction of p130 and p107 appears to play an important role in the MIS-induced inhibition of C33A cell growth (10). On considering the intracellular events, reduction of activity of CDK complexes by CDKIs including p16, p15, p21, and p27 is followed by an increase in the expression of p107 and p130, decrease in E2F activity, and finally, cell cycle arrest $(25,26)$. In the endometrial cancer cell line AN3CA, MIS increases p130 expression and decreases E2F1 expression (11); however, MIS was found to reduce E2F2 and E2F3 expression without altering E2F1 expression. Hence, rather than E2F1, E2F2 and E2F3 are more likely to be associated with the MIS-induced apoptosis of HPV16-related cervical cancer cells. These findings imply that MIS-induced changes in the expression of genes related to cell cycle control and apoptosis in CaSki cells differ from those in other cell lines, just as gene expression changes in cervical cancer cells may vary depending on the cell line.

Increase in the expression of GADD45A, which is known to be involved in cell cycle arrest, and decrease in the expression of the cyclin B1-encoding gene, which is known to be suppressed by $G A D D 45 A$ induction, are novel findings of the experiment and serve as important clues to the anti-tumor mechanism of MIS in HPV16-positive cervical cancer cells. In addition, it is also well known that cytochrome $\mathrm{C}$ is released during apoptosis in small amounts, but not enough to support the hypothesis that apoptosis is the main anti-tumor mechanism of MIS. However, MIS definitely induces apoptosis in CaSki cells as inferred from the apparent increase in apoptosis-related genes such as caspase 3 after MIS treatment.

Therefore, we may think that the major mechanism by which MIS inhibits cell growth in CaSki cells is as follows. MIS suppresses cell division by inducing an increase in the expression of CDKIs such as p16; this results in a decrease in the CDK complex activity, and finally blocks E2F activity. Although the suppression of transition to the $S$ phase by a 
concerted change in the expression of genes responsible for the $\mathrm{G}_{1} / \mathrm{S}$ checkpoint is one of the probable mechanisms, the low levels of $\mathrm{Rb}$ gene expression indicate that it is not the main mechanism.

The E2F family of transcription factors consists of 9 members that are critical to oncogenesis because these factors play a central role in cell growth and proliferation via their ability to regulate genes involved in the cell cycle. In a recent experiment, the overexpressed E2F1 through 6 in non-transformed 3T3 fibroblasts were used to evaluate the ability of these transgenic cell lines to grow under low serum conditions. Those results showed that these 6 E2F family members can be divided into 3 groups that exhibit differential oncogenic capacity. The first group consists of E2F2 and E2F3a, both of which have strong oncogenic capacity as observed in our experiment. The second group consists of E2F1 and E2F6, which were neutral when compared to control cells transfected with the vector alone. The third group consists of E2F4 and E2F5, which generally repress E2F-responsive genes and demonstrated a strong capacity to inhibit transformation in growth inhibition assays. These results imply that the pattern of expression of these 6 E2F family members in a cell could strongly influence the cell's susceptibility to oncogenic transformation E2F1 and E2F6 had weak or no oncogenic capacity. E2F6 is unique in that it retains the conserved E2F DNA binding and dimerization domains but lacks the C-terminal transactivation and pocket protein binding domains characteristic of other members. Therefore, E2F6 can act as a competitive inhibitor of other E2F proteins in DNA binding, and when overexpressed, can oppose the function of both the oncogenic proteins E2F2 and E2F3a as well as the anti-oncogenic protein E2F4 and 5 E2F family members. This behavior may explain why E2F6 is neutral in our transformation assays (27).

This study provides only limited data interpretation on the mechanism by which MIS suppresses HPV-related cervical cancer cells but it does provide a large amount of new genetic information. However, a MIS-derived regimen is possibly advantageous as a curative or palliative treatment option because no specific toxicity is reported to be associated with MIS concentrations as high as 60 times that in a normal newborn male baby and as 1,000 times in patients with granulosa cell tumor (28).

\section{References}

1. MacLaughlin DT and Donahoe PK: Müllerian inhibiting substance: a potential nontoxic, naturally occurring therapeutic agent for certain human cancers. In: Transforming Growth Factor-beta in Cancer Therapy. Jakowlew S (ed). Humana Press, Totowa, NJ, pp333-353, 2008.

2. Lasala C, Carre-Eusebe D, Picard JY and Rey R: Subcellular and molecular mechanisms regulating anti-Müllerian hormone gene expression in mammalian and nonmammalian species. DNA Cell Biol 23: 572-585, 2004.

3. Laurich VM, Trbovich AM, O'Neill FH, et al: Müllerian inhibiting substance blocks the protein kinase A-induced expression of cytochrome P450 17alpha-hydroxylase/C17-20 lyase mRNA in a mouse Leydig cell line independent of cAMP responsive element binding protein phosphorylation. Endocrinology 143: 3351-3360, 2002.

4. Sriraman V, Niu E, Matias JR, et al: Müllerian inhibiting substance inhibits testosterone synthesis in adult rats. J Androl 22: 750-758, 2001.
5. Ha TU, Segev DL, Barbie D, et al: Müllerian inhibiting substance inhibits ovarian cell growth through an $\mathrm{pRb}$-independent mechanism. J Biol Chem 275: 37101-37109, 2000.

6. Chin T, Parry RL and Donahoe PK: Human Müllerian inhibiting substance inhibits tumor growth in vitro and in vivo. Cancer Res 51: 2101-2106, 1991

7. Masiakos PT, MacLaughlin DT, Maheswaran S, et al: Human ovarian cancer, cell lines, and primary ascites cells express the human Müllerian inhibiting substance (MIS) type II receptor, bind, and are responsive to MIS. Clin Cancer Res 5: 3488-3499, 1999.

8. Stephen AE, Pearsall LA, Christian BP, Donahoe PK, Vacanti JP and MacLaughlin DT: Highly purified Müllerian inhibiting substance inhibits human ovarian cancer in vivo. Clin Cancer Res 8: 2640-2646, 2002.

9. Segev DL, Ha TU, Tran TT, et al: Müllerian inhibiting substance inhibits breast cancer cell growth through an $\mathrm{NF} \kappa \mathrm{B}$-mediated pathway. J Biol Chem 275: 28371-28379, 2000.

10. Barbie TU, Barbie DA, MacLaughlin DT, Maheswaran S and Donahoe PK: Müllerian inhibiting substance inhibits cervical cancer cell growth via a pathway involving p130 and p107. Proc Natl Acad Sci USA 100: 15601-15606, 2003.

11. Renaud EJ, MacLaughlin DT, Oliva E, Rueda BR and Donahoe PK: Endometrial cancer is a receptor-mediated target for Müllerian inhibiting substance. Proc Natl Acad Sci USA 102: 111-116, 2005.

12. Imbeaud S, Faure E, Lamarre I, et al: Insensitivity to anti-Müllerian hormone due to a mutation in the human anti-Müllerian hormone receptor. Nat Genet 11: 382-388, 1995.

13. Segev DL, Hoshiya Y, Hoshiya M, et al: Müllerian-inhibiting substance regulates NF- $\kappa$ B signaling in the prostate in vitro and in vivo. Proc Natl Acad Sci USA 99: 239-244, 2002.

14. Josso N and Di Clemente N: Transduction pathway of antiMüllerian hormone, a sex-specific member of the TGF-beta family. Trends Endocrinol Metab 14: 91-97, 2003.

15. Golub TR, Slonim DK, Tamayo P, et al: Molecular classification of cancer: class discovery and class prediction by gene expression monitoring. Science 286: 531-537, 1999.

16. Sørlie T, Perou CM, Tibshirani R, et al: Gene expression patterns of breast carcinomas distinguish tumor subclasses with clinical implications. Proc Natl Acad Sci USA 98: 10869-10874, 2001.

17. Nam SW, Park JY, Ramasamy A, et al: Molecular changes from dysplastic nodule to hepatocellular carcinoma through gene expression profiling. Hepatology 42: 809-818, 2005.

18. Lorenzo HK, Teixeira J, Pahlavan N, Laurich VM, Donahoe PK and MacLaughlin DT: New approaches for high-yield purification of Müllerian inhibiting substance improve its bioactivity. J Chromatogr B Analyt Technol Biomed Life Sci 766: 89-98, 2002.

19. Nam SW, Lee JH, Noh JH, et al: Comparative analysis of expression profiling of early-stage carcinogenesis using nodule-in-noduletype hepatocellular carcinoma. Eur J Gastroenterol Hepatol 18: 239-247, 2006.

20. Noh JH, Ryu SY, Eun JW, et al: Identification of large-scale molecular changes of Autotaxin(ENPP2) knock-down by small interfering RNA in breast cancer cells. Mol Cell Biochem 288: 91-106, 2006

21. Moody CA and Laimins LA: Human papillomavirus oncoproteins: pathways to transformation. Nat Rev Cancer 10: 550-560, 2010.

22. Klaes R, Friedric T, Spitkovsky D, et al: Overexpression of p16 (INK4A) as a specific marker for dysplastic and neoplastic epithelial cells of the cervix uteri. Int J Cancer 92: 276-284, 2001.

23. Pieretti-Vanmarcke R, Donahoe PK, Pearsall LA, et al: Müllerian inhibiting substance enhances subclinical doses of chemotherapeutic agents to inhibit human and mouse ovarian cancer. Proc Natl Acad Sci USA 103: 17426-17431, 2006.

24. MacLaughlin DT and Donahoe PK: Müllerian inhibiting substance/ anti-Müllerian hormone: a potential therapeutic agent for human ovarian and other cancers. Future Oncol 6: 391-405, 2010.

25. Sherr CJ and Roberts JM: Inhibitors of mammalian G1 cyclindependent kinases. Genes Dev 9: 1149-1163, 1995.

26. Hirai H, Roussel MF, Kato JY, Ashmun RA and Sherr CJ: Novel INK4 proteins, p19 and p18, are specific inhibitors of the cyclin Ddependent kinases CDK4 and CDK6. Mol Cell Biol 15: 2672-2681, 1995.

27. Chen $\mathrm{C}$ and Wells AD: Comparative analysis of E2F family member oncogenic activity. PLoS One 2: e912, 2007.

28. Pieretti-Vanmarcke R, Donahoe PK, Szotek P, et al: Recombinant human Müllerian inhibiting substance inhibits long-term growth of MIS type II receptor-directed transgenic mouse ovarian cancer in vivo. Clin Cancer Res 12: 1593-1598, 2006. 